\title{
DIRICHLET PROCESSES AND AN INTRINSIC CHARACTERIZATION OF RENORMALIZED INTERSECTION LOCAL TIMES
}

\author{
Jay ROSEN ${ }^{1}$ \\ Department of Mathematic, College of Staten Island, CUNY, Staten Island, NY 10314, USA
}

Received 15 June 1999, revised 28 August 2000

ABSTRACT. - We show that the $n$th order renormalized self-intersection local time $\gamma_{n}(\mu ; t)$ for the symmetric stable process in $R^{2}$, where the $n$-fold multiple points are weighted by an arbitrary measure $\mu$, can be characterized as the continuous process of zero quadratic variation in the decomposition of a natural Dirichlet process. This Dirichlet process is the potential of a random measure associated with $\gamma_{n-1}(\mu ; t)$. ๑ 2001 Éditions scientifiques et médicales Elsevier SAS

RÉSUMÉ. - Lorsque les points multiples d'arche $n$ sont changés par une mesure arbitraire $\mu$, le temps local d'auto-intersection renormalisé $\gamma_{n}(\mu, t)$ du processus symétrique stable de $R^{2}$ peutêtre caractérisé comme le processus de variation de quadratique nulle de la décomposition d'un processus de Dirichlet défini naturellement comme le potentiel d'une mesure aléatoire associée à $\gamma_{n-1}(\mu, t)$. @ 2001 Éditions scientifiques et médicales Elsevier SAS

\section{Introduction}

Renormalized intersection local time can be thought of as an attempt to "measure" the amount of self-intersections of a stochastic process, say, $X(t) \in R^{d}$. A natural approach is to set

$$
\alpha_{2, \varepsilon}(\mu, t) \stackrel{\text { def }}{=} \iint_{\left\{0 \leqslant t_{1} \leqslant t_{2} \leqslant t\right\}} f_{\varepsilon}\left(X_{t_{1}}-x\right) f_{\varepsilon}\left(X_{t_{2}}-X_{t_{1}}\right) \mathrm{d} t_{1} \mathrm{~d} t_{2} \mathrm{~d} \mu(x)
$$

where $f_{\varepsilon}$ is an approximate $\delta$-function at zero, and take the limit as $\varepsilon \rightarrow 0$. Intuitively, this gives a measure of the set of times $\left(t_{1}, t_{2}\right)$ such that $X_{t_{1}}=X_{t_{2}}=x$, where the "double points" $x \in R^{d}$ are weighted by the measure $\mu$. However, in general, this limit does not exist because of the effect of the integral in the neighborhood of the diagonal. The method used to compensate for this is called renormalization. For simplicity we restrict

E-mail address: jrosen3@earthlink.net (J. Rosen).

${ }^{1}$ This research was supported, in part, by grants from the National Science Foundation and PSC-CUNY. 
our discussion to symmetric Lévy processes. The renormalized intersection local time $\gamma_{2}(\mu, t)$ is defined as the $\varepsilon \rightarrow 0$ limit of

$$
\gamma_{2, \varepsilon}(\mu, t)=\alpha_{2, \varepsilon}(\mu, t)-u_{\varepsilon}^{1}(0) \int_{0}^{t} f_{\varepsilon}\left(X_{t_{1}}-x\right) \mathrm{d} t_{1} \mathrm{~d} \mu(x)
$$

whenever the limit exists. Here $u_{\varepsilon}^{1}(x)=\int f_{\varepsilon}(x-y) u^{1}(y) \mathrm{d} y$ and $u^{1}(x)=\int_{0}^{\infty} \mathrm{e}^{-t} p_{t}(x) \mathrm{d} t$ is the 1-potential density. In general, $u_{\varepsilon}^{1}(0) \uparrow \infty$. In the most studied case, $\mu$ is taken to be Lebesgue measure so that the subtraction term in (1.2) is simply $u_{\varepsilon}^{1}(0) t . \gamma_{2}(\mu, t)$ is an interesting new functional in its own right, but the fact that it is obtained from a divergent functional by subtracting off an infinite counterterm leaves the 'meaning' of $\gamma_{2}(\mu, t)$ rather puzzling. This puzzle is only deepened when we find that renormalized intersection local time, originally studied by Varadhan [16] for its role in quantum field theory, turns out to be the right tool for the solution of certain "classical" problems such as the asymptotic expansion of the area of the Wiener and stable sausage in the plane and fluctuations of the range of stable random walks. (See Le Gall [8,7], Le Gall and Rosen [10] and Rosen [14]. For further work on renormalized intersection local times see Dynkin [4], Le Gall [9], Bass and Khoshnevisan [1], Rosen [15] and Marcus and Rosen [11]). When we turn to the higher order renormalized intersection local time $\gamma_{n}(\mu, t)$ with its complicated scheme of subtractions, see (1.3), the need to find the intrinsic meaning' of $\gamma_{n}(\mu, t)$ becomes pressing. $\gamma_{n}(\mu, t)$ is defined as the $\varepsilon \rightarrow 0$ limit of

$$
\gamma_{n, \varepsilon}(\mu, t)=\sum_{k=0}^{n-1}(-1)^{k}\left(\begin{array}{c}
n-1 \\
k
\end{array}\right)\left(u_{\varepsilon}^{1}(0)\right)^{k} \alpha_{n-k, \varepsilon}(\mu, t),
$$

where

$$
\alpha_{n, \varepsilon}(\mu, t) \stackrel{\text { def }}{=} \iint_{\left\{0 \leqslant t_{1} \leqslant \cdots \leqslant t_{n} \leqslant t\right\}} f_{\varepsilon}\left(X_{t_{1}}-x\right) \prod_{j=2}^{n} f_{\varepsilon}\left(X_{t_{j}}-X_{t_{j-1}}\right) \mathrm{d} t_{1} \cdots \mathrm{d} t_{n} \mathrm{~d} \mu(x)
$$

measures of the set of times $\left(t_{1}, \ldots, t_{n}\right)$ such that $X_{t_{1}}=\cdots=X_{t_{n}}=x$, with the " $n$ multiple points" $x \in R^{d}$ weighted by the measure $\mu$.

The goal of this paper is to provide a natural characterization for $\gamma_{n}(\mu, t)$ which we expect will lead to a deeper understanding of this important functional. The key idea is that $\gamma_{n}(\mu, t)$ has zero quadratic variation. In the case of second order renormalized intersection local time $\gamma_{2}(\mu, t)$ for Brownian motion in the plane, with $\mu$ taken to be Lebesgue measure, this was observed by Bertoin [2].

Recall that a continuous adapted process $Z_{t}$ is said to have zero quadratic variation, if for each $T>0$ and any sequence of partitions $\tau_{n}=\left\{0=t_{0}<t_{1}<\cdots<t_{n}=T\right\}$ of $[0, T]$, with mesh size $\left|\tau_{n}\right|=\max _{i}\left|t_{i}-t_{i-1}\right|$ going to 0

$$
\lim _{n \rightarrow \infty} E\left(\sum_{t_{i} \in \tau_{n}}\left(Z_{t_{i}}-Z_{t_{i-1}}\right)^{2}\right)=0 .
$$

Föllmer, [6] has coined the term "Dirichlet process" to refer to any process which can be written as the sum of a martingale and a process of zero quadratic variation. It is 
important to note that such a decomposition is unique. The class of Dirichlet processes is much wider than the class of semimartingales.

In the following we use $Y_{t}$ to denote our $R^{d}$ valued Lévy process $X_{t}$ killed at an independent exponential time $\lambda$. In the following theorems the renormalized intersection local times $\gamma_{k}(\mu ; t)$ will be defined for the process $Y_{t}$ in place of $X_{t}$.

Let us begin with a special case of the Doob-Meyer decomposition for semimartingales. Let $L_{t}^{\mu}$ denotes the continuous additive functional of $X_{t}$ with Revuz measure $\mu$. Using the additivity of $L_{t}^{\mu}$ and the Markov property we have

$$
E^{x}\left(L_{\lambda}^{\mu} \mid \mathcal{F}_{t}\right)=L_{t \wedge \lambda}^{\mu}+U^{1} \mu\left(Y_{t}\right)
$$

where $\mathcal{F}_{t}=\sigma\left(Y_{s}, s \leqslant t\right)$. Equivalently, $U^{1} \mu\left(Y_{t}\right)=M_{t}-L_{t \wedge \lambda}^{\mu}$ where $M_{t}=E^{x}\left(L_{\lambda}^{\mu} \mid \mathcal{F}_{t}\right)$ is a martingale. This is the Doob-Meyer decomposition for the potential $U^{1} \mu\left(Y_{t}\right)$. We will show that $\gamma_{2}(\mu, t)$ arises in a similar decomposition for the potential of a random measure. This new potential will no longer be a semimartingale but a Dirichlet process, and $\gamma_{2}(\mu, t)$ will correspond to the process of zero quadratic variation in the decomposition of this Dirichlet process.

Let $\pi_{t}^{\mu}$ be the random additive measure-valued process defined by

$$
\pi_{t}^{\mu}(A)=L_{t}^{1_{A} \cdot \mu}=\int_{0}^{t} 1_{A}\left(Y_{s}\right) \mathrm{d} L_{s}^{\mu}
$$

for all $A \subseteq R^{d}$. Note that

$$
U^{1} \pi_{t}^{\mu}\left(Y_{t}\right)=\int_{0}^{t} u^{1}\left(Y_{t}-Y_{s}\right) \mathrm{d} L_{s}^{\mu} .
$$

When $\mu$ is Lebesgue measure, so that $L_{s}^{\mu}=s$, we simply write $\gamma_{2}(t)$ and $\pi_{t}$ for $\gamma_{2}(\mu, t)$ and $\pi_{t}^{\mu}$. Thus, $\pi_{t}(A)=\int_{0}^{t} 1_{A}\left(Y_{s}\right) \mathrm{d} s$. We have the following analogue of the DoobMeyer decomposition.

THEOREM 1. - Let $Y$ be a symmetric stable process of order $\beta>4 / 3$ in $R^{2}$, killed at an independent exponential time $\lambda$. Then $\gamma_{2}(t)$ is continuous a.s. with zero quadratic variation and

$$
U^{1} \pi_{t}\left(Y_{t}\right)=M_{t}-\gamma_{2}(t)
$$

where $M_{t}$ is the martingale $E^{x}\left(\gamma_{2}(\lambda) \mid \mathcal{F}_{t}\right)$.

In view of Theorem 1 we can characterize the renormalized intersection local time $\gamma_{2}(t)$ as the continuous process of zero quadratic variation in the decomposition of the random potential $U^{1} \pi_{t}\left(Y_{t}\right)=\int_{0}^{t} u^{1}\left(Y_{t}-Y_{s}\right) \mathrm{d} s$. (Our approach is different from Bertoin's. He uses stochastic calculus, which essentially restricts one to the study of Brownian motion.)

Theorem 1 is an immediate consequence of the next Theorem for general $\mu$. We let $D(x, \varepsilon) \subseteq R^{2}$ denote the disc of radius $\varepsilon$ centered at $x$. 
THEOREM 2. - Let $Y$ be a symmetric stable process of order $1<\beta$ in $R^{2}$, killed at an independent exponential time $\lambda$. If $U^{1} \mu(x)$ is bounded and

$$
\sup _{x} \int_{D(x, 1)} \frac{1}{|x-y|^{3(2-\beta)+\zeta}} \mathrm{d} \mu(y)<\infty
$$

for some $\zeta>0$ with $3(2-\beta)+2 \zeta>2$, then $\gamma_{2}(\mu ; t)$ is continuous a.s. with zero quadratic variation and

$$
U^{1} \pi_{t}^{\mu}\left(Y_{t}\right)=M_{t}-\gamma_{2}(\mu, t)
$$

where $M_{t}$ is the martingale $E^{x}\left(\gamma_{2}(\mu, \lambda) \mid \mathcal{F}_{t}\right)$.

Note in particular that for Brownian motion, condition (1.10) is the condition that

$$
\sup _{x} \int_{D(x, 1)} \frac{1}{|x-y|^{\zeta}} \mathrm{d} \mu(y)<\infty
$$

for some $\zeta>1$.

For each $A \in \mathcal{B}\left(R^{2}\right)$, the Borel sets in $R^{2}$, let us define

$$
\pi_{t}^{\mu, n-1}(A)=\gamma_{n-1}\left(1_{A} \cdot \mu, t\right)
$$

If $\pi_{t}^{\mu, n-1}(A)$ is a (random) measure in $A$, we use $U^{1} \pi_{t}^{\mu, n-1}$ to denote its potential.

The following Theorem leads inductively to an intrinsic characterization of the higher order renormalized intersection local times $\gamma_{n}(\mu, t)$ which does not use limiting procedures.

THEOREM 3. - Let $Y$ be a symmetric stable process of order $\beta>1$ in $R^{2}$, killed at an independent exponential time $\lambda$. If $U^{1} \mu(x)$ is bounded and

$$
\sup _{x} \int_{D(x, 1)} \frac{1}{|x-y|^{(2 n-1)(2-\beta)+\zeta}} \mathrm{d} \mu(y)<\infty
$$

for some $\zeta>0$ with $(2 n-1)(2-\beta)+2 \zeta>2$, then $\gamma_{n}(\mu ; t)$ is continuous a.s. with zero quadratic variation and we can choose a version of $\left\{\pi_{t}^{\mu, n-1}(A) ;(A, t) \in \mathcal{B}\left(R^{2}\right) \times R_{+}\right\}$ which is a.s. a measure in $A$ and continuous in $t$, and such that

$$
U^{1} \pi_{t}^{\mu, n-1}\left(Y_{t}\right)=M_{t}-\gamma_{n}(\mu, t)
$$

where $M_{t}$ is the martingale $E^{x}\left(\gamma_{n}(\mu, \lambda) \mid \mathcal{F}_{t}\right)$.

Remarks. - (i) For Brownian motion, condition (1.14) reduces to the condition (1.12) for some $\zeta>1$.

(ii) As before, when $\mu$ is Lebesgue measure we simply write $\gamma_{n}(t)$ and $\pi_{t}^{n}$ for $\gamma_{n}(\mu, t)$ and $\pi_{t}^{\mu, n}$. The following is an immediate consequence of Theorem 3 . 
COROLlaRY 1. - Let $Y$ be a symmetric stable process in $R^{2}$ of order $\beta>2(2 n-$ $2) /(2 n-1)$ killed at an independent exponential time $\lambda$. Then $\gamma_{n}(t)$ is continuous a.s. with zero quadratic variation and we can choose a version of $\left\{\pi_{t}^{n-1}(A) ;(A, t) \in\right.$ $\left.\mathcal{B}\left(R^{2}\right) \times R_{+}\right\}$which is a.s. a measure in $A$ and continuous in $t$, and such that

$$
U^{1} \pi_{t}^{n-1}\left(Y_{t}\right)=M_{t}-\gamma_{n}(t)
$$

where $M_{t}$ is the martingale $E^{x}\left(\gamma_{n}(\lambda) \mid \mathcal{F}_{t}\right)$.

Note in particular that for Brownian motion our Theorem holds for all $n$.

(iii) The necessary and sufficient condition for the existence of $n$-multiple points is that $\int\left(u^{1}(y)\right)^{n} \mathrm{~d} y<\infty$, see [5]. In [11] we show that

$$
E\left(\left\{\gamma_{n}(\mu ; t)\right\}^{2}\right)=\iint u^{1}(x)\left(u^{1}(x-y)\right)^{2 n-1} \mathrm{~d} \mu(x) \mathrm{d} \mu(y) .
$$

Since $u^{1}(x) \sim C x^{-(2-\beta)}$ as $|x| \rightarrow 0,(1.14)$ is natural. In fact, $U^{1} \mu(x)$ bounded and (1.14) are precisely the conditions required by the techniques of [15] to show that $\gamma_{n}(\mu ; t)$ is continuous a.s.

(iv) The representation (1.15) is obtained in [12]. The new element here is that $\gamma_{n}(\mu ; t)$ is continuous a.s. with zero quadratic variation

Of course, Theorem 2 is a special case of Theorem 3. We expect that the situation is very different for processes in $R^{3}$. Recall that when $\mu$ is Lebesgue measure, (1.7) has the particularly simple form: $\pi_{t}^{\mu}(A)=\int_{0}^{t} 1_{A}\left(Y_{s}\right) \mathrm{d} s$. This is certainly well defined when $Y_{s}$ is Brownian motion in $R^{3}$. We expect that in this case the random potential $U^{1} \pi_{t}^{\mu}\left(Y_{t}\right)$ is not a Dirichlet process, in contradistinction to the situation in $R^{2}$. We are led to this conjecture by the fact that the renormalized intersection local time $\gamma_{2}(\mu, t)$ does not exist for Brownian motion in $R^{3}$, [13].

For the remainder of this paper we fix a symmetric stable process of order $\beta>1$ in $R^{2}$ and use the notation $v=2 / \beta-1$.

This paper is organized as follows. In Section 2 we develop a new representation for $E\left(\gamma_{n}^{2}(\mu ; t)\right)$, which is then used in Sections 3 and 4 to prove Theorem 3. As mentioned, this implies Theorems 1-2. In the final section we prove some estimates used in the proof of Theorem 3.

\section{A new representation for $E\left(\gamma_{n}^{2}(\mu ; t)\right)$}

Recall that $u_{\varepsilon}^{1}(x)=\int f_{\varepsilon}(x-y) u^{1}(y) \mathrm{d} y$ and $u^{1}(x)=\int_{0}^{\infty} \mathrm{e}^{-t} p_{t}(x) \mathrm{d} t$ is the 1-potential density of the unkilled process. We will use $q_{t}(x)$ for the transition density for our exponentialy killed process and set $q_{t, \varepsilon}(x)=\int f_{\varepsilon}(x-y) q_{t}(y) \mathrm{d} y$. Our starting point is the following formula which comes from the proof of Lemma 7 of [12]:

$$
\begin{aligned}
E\left(\gamma_{n}^{2}(\mu ; t)\right)= & \lim _{\varepsilon \rightarrow 0} \sum_{s \in \mathcal{S}} \iiint_{\left\{\sum t_{p} \leqslant t\right\}} \prod_{p \in B_{s}}\left\{q_{t_{p}, \varepsilon}(0)-u_{\varepsilon}^{1}(0) \delta\left(t_{p}\right)\right\} \prod_{p \in B_{s}^{c}} q_{t_{p}}(x-y) \\
& \times \prod_{p} \mathrm{~d} t_{p} \mathrm{~d} \mu(x) \mathrm{d} \mu(y)
\end{aligned}
$$




$$
\begin{aligned}
= & \lim _{\varepsilon \rightarrow 0} \sum_{s \in \mathcal{S}} \iiint_{\left\{0 \leqslant t_{1} \leqslant \cdots \leqslant t_{2 n} \leqslant t\right\}} \prod_{p \in B_{s}}\left\{q_{t_{p}-t_{p-1}, \varepsilon}(0)-u_{\varepsilon}^{1}(0) \delta\left(t_{p}-t_{p-1}\right)\right\} \\
& \times \prod_{p \in B_{s}^{c}} q_{t_{p}-t_{p-1}}(x-y) \prod_{p} \mathrm{~d} t_{p} \mathrm{~d} \mu(x) \mathrm{d} \mu(y),
\end{aligned}
$$

where $\mathcal{S}$ is the set of mappings

$$
s:\{1, \ldots, 2 n\} \mapsto\{1,2\}
$$

such that $\left|s^{-1}(i)\right|=n, i=1,2, B_{s}=\{p \mid s(p)=s(p-1)\}$.

We intend to rewrite this in a form in which the $u_{\varepsilon}^{1}(0) \delta\left(t_{p}-t_{p-1}\right)$ terms have been eliminated. To this end we define several operators on $\mathcal{C}(0, t]$ the space of continuous functions on $(0, t]$ :

$$
\begin{gathered}
I_{\uparrow}^{\varepsilon}(h)(r)=\left(\int_{r}^{\infty} q_{s, \varepsilon}(0) \mathrm{d} s\right) h(r)=Q_{\varepsilon}(r) h(r), \\
I_{\downarrow}^{\varepsilon}(h)(r)=\int_{0}^{r} q_{s, \varepsilon}(0) \mathcal{D}_{-s} h(r-s) \mathrm{d} s, \\
J_{1}^{\varepsilon}(h)(r)=-I_{\uparrow}^{\varepsilon}(h)(r)+I_{\downarrow}^{\varepsilon}(h)(r),
\end{gathered}
$$

and

$$
J_{2}^{\varepsilon}(h)(r)=\int_{0}^{r} q_{s}(x-y) h(r-s) \mathrm{d} s .
$$

Here, $\mathcal{D}_{x}$ applied to a function of the variable $x$ is defined by $\mathcal{D}_{x} f=f(x)-f(0)$, and although $J_{2}^{\varepsilon}(h)(r)$ doesn't depend on $\varepsilon$, we have included the $\varepsilon$ in $J_{2}^{\varepsilon}(h)(r)$ for notational convienience.

We will show how to express each summand on the r.h.s. of (2.1) in terms of the operators $J_{1}^{\varepsilon}, J_{2}^{\varepsilon}$. This representation will be used to show that $\gamma_{n}(\mu ; t)$ has 0 quadratic variation.

Define $r_{s}(p)=1$ if $p \in B_{s}$ and $r_{s}(p)=2$ if $p \in B_{s}^{c}$. Then using the notation $J_{a}^{\varepsilon} J_{b}^{\varepsilon}$ for the iteration of the operators $J_{a}^{\varepsilon}$ and $J_{b}^{\varepsilon}$ have

LEMMA 1.-

$$
E\left(\gamma_{n}^{2}(\mu ; t)\right)=\lim _{\varepsilon \rightarrow 0} \sum_{s \in \mathcal{S}} \iiint_{0}^{t} q_{s_{1}}(x) J_{r_{s}(2)}^{\varepsilon} J_{r_{s}(3)}^{\varepsilon} \cdots J_{r_{s}(2 n)}^{\varepsilon}(1)\left(t-s_{1}\right) \mathrm{d} s_{1} \mathrm{~d} \mu(x) \mathrm{d} \mu(y) .
$$

Proof. - Our approach consists of taking the right hand side of (2.1), integrating $\mathrm{d} t_{i}$ in decreasing order of $i$, and rewriting the resulting integrals. Fix $s \in \mathcal{S}$.

When $i \in B_{s}$ we use: 


$$
\begin{aligned}
\int_{t_{i-1}}^{t}\{ & \left.q_{t_{i}-t_{i-1}, \varepsilon}(0)-u_{\varepsilon}^{1}(0) \delta\left(t_{i}-t_{i-1}\right)\right\} h\left(t-t_{i}\right) \mathrm{d} t_{i} \\
& =\int_{t_{i-1}}^{t} q_{t_{i}-t_{i-1}, \varepsilon}(0) h\left(t-t_{i}\right) \mathrm{d} t_{i}-u_{\varepsilon}^{1}(0) h\left(t-t_{i-1}\right) \\
= & \int_{0}^{t-t_{i-1}} q_{s, \varepsilon}(0) h\left(t-t_{i-1}-s\right) \mathrm{d} s-u_{\varepsilon}^{1}(0) h\left(t-t_{i-1}\right) \\
= & \int_{0}^{t-t_{i-1}} q_{s, \varepsilon}(0) h\left(t-t_{i-1}\right) \mathrm{d} s-u_{\varepsilon}^{1}(0) h\left(t-t_{i-1}\right) \\
& +\int_{0}^{t-t_{i-1}} q_{s, \varepsilon}(0) \mathcal{D}_{-s} h\left(t-t_{i-1}-s\right) \mathrm{d} s \\
= & \left(\int_{0}^{t-t_{i-1}} q_{s, \varepsilon}(0) \mathrm{d} s-u_{\varepsilon}^{1}(0)\right) h\left(t-t_{i-1}\right)+I_{\downarrow}^{\varepsilon}(h)\left(t-t_{i-1}\right) \\
= & J_{1}^{\varepsilon}(h)\left(t-t_{i-1}\right) .
\end{aligned}
$$

While if $i \in B_{s}^{c}$ we use:

$$
\begin{aligned}
\int_{t_{i-1}}^{t} q_{t_{i}-t_{i-1}}(x-y) h\left(t-t_{i}\right) \mathrm{d} t_{i} & =\int_{0}^{t-t_{i-1}} q_{s}(x-y) h\left(t-t_{i-1}-s\right) \mathrm{d} s \\
& =J_{2}^{\varepsilon}(h)\left(t-t_{i-1}\right) .
\end{aligned}
$$

This completes the proof of Lemma 1.

To show that $\gamma_{n}(\mu ; t)$ has 0 quadratic variation we will need the following modification of Lemma 1. For a fixed $s \in \mathcal{S}$, let $\bar{p}(s)=\max \{p \mid s(p) \neq s(2 n)\}$.

LEMMA 2. - For any $r_{i-1}<r_{i}$

$$
\begin{aligned}
E\left(\left\{\gamma_{n}\left(\mu ; r_{i}\right)-\gamma_{n}\left(\mu ; r_{i-1}\right)\right\}^{2}\right) \\
=\lim _{\varepsilon \rightarrow 0} \sum_{s \in \mathcal{S}} \iiint_{r_{i-1}}^{r_{i}} q_{s_{1}}(x) J_{r_{s}(2)}^{\varepsilon} J_{r_{s}(3)}^{\varepsilon} \cdots J_{r_{s}(2 n)}^{\varepsilon}(1)\left(r_{i}-s_{1}\right) \mathrm{d} s_{1} \mathrm{~d} \mu(x) \mathrm{d} \mu(y) \\
\quad+\sum_{j=2}^{\bar{p}(s)} \iiint_{0}^{r_{i-1}} q_{s_{1}}(x) J_{r_{s}(2)}^{\varepsilon} J_{r_{s}(3)}^{\varepsilon} \cdots \bar{J}_{r_{s}(j)}^{\varepsilon} \cdots \\
\quad \times J_{r_{s}(2 n)}^{\varepsilon}(1)\left(r_{i-1}-s_{1}\right) \mathrm{d} s_{1} \mathrm{~d} \mu(x) \mathrm{d} \mu(y)
\end{aligned}
$$


where

$$
\bar{J}_{1}^{\varepsilon}(h)(s)=\int_{r_{i-1}}^{r_{i}} q_{t-s, \varepsilon}(0) h\left(r_{i}-t\right) \mathrm{d} t
$$

and

$$
\bar{J}_{2}^{\varepsilon}(h)(s)=\int_{r_{i-1}}^{r_{i}} q_{t-s}(x-y) h\left(r_{i}-t\right) \mathrm{d} t .
$$

Proof. -

As in (2.1),

$$
\begin{aligned}
& E\left(\left\{\gamma_{n}\left(\mu ; r_{i}\right)-\gamma_{n}\left(\mu ; r_{i-1}\right)\right\}^{2}\right) \\
& =\lim _{\varepsilon \rightarrow 0} \sum_{s \in \mathcal{S}} \iiint_{\substack{\left\{0 \leqslant t_{1} \leqslant \cdots \leqslant t_{2 n} \leqslant r_{i}\right\} \\
\left\{r_{i-1}<t_{\bar{p}(s)} \leqslant t_{2 n} \leqslant r_{i}\right\}}} \prod_{p \in B_{s}}\left\{q_{t_{p}-t_{p-1}, \varepsilon}(0)-u_{\varepsilon}^{1}(0) \delta\left(t_{p}-t_{p-1}\right)\right\} \\
& \quad \times \prod_{p \in B_{s}^{c}} q_{t_{p}-t_{p-1}}(x-y) \prod_{p} \mathrm{~d} t_{p} \mathrm{~d} \mu(x) \mathrm{d} \mu(y) .
\end{aligned}
$$

Here we have used the $\delta\left(t_{p}-t_{p-1}\right)$ notation to allow us to write the condition arising in the quadratic variation in the compact form $r_{i-1}<t_{\bar{p}(s)} \leqslant t_{2 n} \leqslant r_{i}$.

We have

$$
\begin{aligned}
\{0 & \left.\leqslant t_{1} \leqslant \cdots \leqslant t_{2 n} \leqslant r_{i} ; r_{i-1}<t_{\bar{p}(s)} \leqslant t_{2 n} \leqslant r_{i}\right\} \\
& =\left\{0 \leqslant t_{1} \leqslant \cdots \leqslant t_{2 n} \leqslant r_{i} ; r_{i-1}<t_{\bar{p}(s)} \leqslant r_{i}\right\} \\
& =\bigcup_{j=1}^{\bar{p}(s)}\left\{0 \leqslant t_{1} \leqslant \cdots \leqslant t_{j-1} \leqslant r_{i-1}<t_{j} \leqslant \cdots \leqslant t_{2 n} \leqslant r_{i}\right\}
\end{aligned}
$$

where the union is disjoint.

Thus, using the proof of Lemma 1

$$
\begin{aligned}
& \iiint_{\substack{\left\{0 \leqslant t_{1} \leqslant \cdots \leqslant t_{2 n} \leqslant r_{i}\right\} \\
\left\{r_{i-1}<t_{\bar{p}(s)} \leqslant t_{2 n} \leqslant r_{i}\right\}}} \prod_{p \in B_{s}}\left\{q_{t_{p}-t_{p-1}, \varepsilon}(0)-u_{\varepsilon}^{1}(0) \delta\left(t_{p}-t_{p-1}\right)\right\} \\
& \times \prod_{p \in B_{s}^{c}} q_{t_{p}-t_{p-1}}(x-y) \prod_{p} \mathrm{~d} t_{p} \mathrm{~d} \mu(x) \mathrm{d} \mu(y) \\
= & \sum_{j=1} \iiint_{\left\{0 \leqslant t_{1} \leqslant \cdots \leqslant t_{2 n} \leqslant r_{i}\right\}} \prod_{p \in B_{s}}\left\{q_{t_{p}-t_{p-1}, \varepsilon}(0)-u_{\varepsilon}^{1}(0) \delta\left(t_{p}-t_{p-1}\right)\right\} \\
& \times \prod_{\left.p t_{j-1} \leqslant r_{i-1}<t_{j}\right\}} q_{q_{p}-t_{p-1}}(x-y) \prod_{p} \mathrm{~d} t_{p} \mathrm{~d} \mu(x) \mathrm{d} \mu(y) \\
= & \lim _{\varepsilon \rightarrow 0} \sum_{s \in \mathcal{S}} \iint_{r_{i-1}}^{r_{i}} q_{s_{1}}(x) J_{r_{s}(2)}^{\varepsilon} J_{r_{s}(3)}^{\varepsilon} \cdots J_{r_{s}(2 n)}^{\varepsilon}(1)\left(r_{i}-s_{1}\right) \mathrm{d} s_{1} \mathrm{~d} \mu(x) \mathrm{d} \mu(y)
\end{aligned}
$$




$$
+\sum_{j=2}^{\bar{p}(s)} \iiint_{0}^{r_{i-1}} q_{s_{1}}(x) J_{r_{s}(2)}^{\varepsilon} J_{r_{s}(3)}^{\varepsilon} \cdots \bar{J}_{r_{s}(j)}^{\varepsilon} \cdots J_{r_{s}(2 n)}^{\varepsilon}(1)\left(r_{i-1}-s_{1}\right) \mathrm{d} s_{1} \mathrm{~d} \mu(x) \mathrm{d} \mu(y),
$$

where we have used the fact that

$$
\begin{aligned}
& \int_{t_{j-1}}^{r_{i}} 1_{\left\{t_{j-1} \leqslant r_{i-1}<t_{j}\right\}}\left\{q_{t_{j}-t_{j-1}, \varepsilon}(0)-u_{\varepsilon}^{1}(0) \delta\left(t_{j}-t_{j-1}\right)\right\} h\left(r_{i}-t_{j}\right) \mathrm{d} \\
& \quad=1_{\left\{t_{j-1} \leqslant r_{i-1}\right\}} \int_{r_{i-1}}^{r_{i}} q_{t_{j}-t_{j-1}, \varepsilon}(0) h\left(r_{i}-t_{j}\right) \mathrm{d} t_{j}, t_{j}
\end{aligned}
$$

and

$$
\begin{aligned}
& \int_{t_{j-1}}^{r_{i}} 1_{\left\{t_{j-1} \leqslant r_{i-1}<t_{j}\right\}} q_{t_{j}-t_{j-1}}(x-y) h\left(r_{i}-t_{j}\right) \mathrm{d} t_{j} \\
& \quad=1_{\left\{t_{j-1} \leqslant r_{i-1}\right\}} \int_{r_{i-1}}^{r_{i}} q_{t_{j}-t_{j-1}}(x-y) h\left(r_{i}-t_{j}\right) \mathrm{d} t_{j} .
\end{aligned}
$$

and note that we can remove the factor $1_{\left\{t_{j-1} \leqslant r_{i-1}\right\}}$ in these displays, since it is automatically taken into account in the subsequent integration. This completes the proof of Lemma 2.

\section{A uniform upper bound}

In this section we will show that each term on the right hand side of (2.6) is uniformly bounded in $\varepsilon>0$. Although not strictly necessary for proving Theorem 3, this will give us an opportunity to develop, in a simpler setting, the tools and ideas used in our proof that $\gamma_{n}(\mu ; t)$ has 0 quadratic variation. To anticipate things a bit, we mention that the real problem in showing the uniform bound in $\varepsilon>0$ comes from $I_{\downarrow}$, due to the nonintegrability of $q_{s}$ near $s=0$. The remedy will be found in $\mathcal{D}_{-s}$. We now develop this idea in detail.

Let $J_{(1, \uparrow)}^{\varepsilon}=-I_{\uparrow}^{\varepsilon}$ and $J_{(1, \downarrow)}^{\varepsilon}=I_{\downarrow}^{\varepsilon}$. We fix $s \in \mathcal{S}$, and breaking up $J_{1}^{\varepsilon}$ as $J_{(1, \uparrow)}^{\varepsilon}+J_{(1, \downarrow)}^{\varepsilon}$ we can write

$$
\begin{aligned}
& \iiint_{0}^{t} q_{s_{1}}(x) J_{r_{s}(2)}^{\varepsilon} J_{r_{s}(3)}^{\varepsilon} \cdots J_{r_{s}(2 n)}^{\varepsilon}(1)\left(t-s_{1}\right) \mathrm{d} s_{1} \mathrm{~d} \mu(x) \mathrm{d} \mu(y) \\
& =\sum_{U \subseteq B_{s}} \iiint_{0}^{t} q_{s_{1}}(x) J_{r_{s(2), U}}^{\varepsilon} J_{r_{s(3), U}}^{\varepsilon} \cdots J_{r_{s(2 n), U}}^{\varepsilon}(1)\left(t-s_{1}\right) \mathrm{d} s_{1} \mathrm{~d} \mu(x) \mathrm{d} \mu(y),
\end{aligned}
$$

where $r_{s(j), U}=(1, \downarrow)$ if $j \in U, r_{s(j), U}=(1, \uparrow)$ if $j \in V \stackrel{\text { def }}{=} B_{s}-U$, and $r_{s(j), U}=2$ if $j \in B_{s}^{c}$. Fixing $U \subseteq B_{s}$, and setting $W=B_{s}^{c}-\{1\}$ we can rewrite each summand in the right hand side of (3.1): 


$$
\begin{aligned}
\iiint_{0}^{t} q_{s_{1}}(x) J_{r_{s(2), U}}^{\varepsilon} J_{r_{s(3), U}}^{\varepsilon} \cdots J_{r_{s(2 n), U}}^{\varepsilon}(1)\left(t-s_{1}\right) \mathrm{d} s_{1} \mathrm{~d} \mu(x) \mathrm{d} \mu(y) \\
=(-1)^{|V|} \iiint 1_{[0, t]}\left(s_{1}\right) q_{s_{1}}(x) \prod_{i \in U} 1_{[0, t]}\left(\sum_{l \leqslant i} s_{l}\right) q_{s_{i}, \varepsilon}(0) \mathcal{D}_{-s_{i}} \\
\\
\quad \prod_{j \in V} Q_{\varepsilon}\left(t-\sum_{l<j} s_{l}\right) \delta\left(s_{j}\right) \prod_{k \in W} 1_{[0, t]}\left(\sum_{l \leqslant k} s_{l}\right) q_{s_{k}}(y-x) \prod_{l=1}^{2 n} \mathrm{~d} s_{l} \mathrm{~d} \mu(x) \mathrm{d} \mu(y),
\end{aligned}
$$

where it is to be understood that the operator $\mathcal{D}_{-s_{m}}$ operates only on the factors $1_{[0, t]}\left(\sum_{l \leqslant i} s_{l}\right), Q_{\varepsilon}\left(t-\sum_{l<j} s_{l}\right)$ and $1_{[0, t]}\left(\sum_{l \leqslant k} s_{l}\right)$ corresponding to $i, j, k>m$. We note that the many factors $1_{[0, t]}\left(\sum_{l \leqslant i} s_{l}\right)$ are redundant, but keeping them will make the connection with the following section, where we prove Theorem 3, easier.

We then use the fact that $\mathcal{D}_{x} f g=\left(\mathcal{D}_{x} f\right) g(x)+f(0) \mathcal{D}_{x} g$ to rewrite the right hand side of (3.3) as a sum of many terms, in which each $\mathcal{D}_{-s_{m}}$ is applied to one factor of the form $1_{[0, t]}\left(\sum_{l \leqslant j} s_{l}\right)$ or $Q_{\varepsilon}\left(t-\sum_{l<j} s_{l}\right)$ with $j>m$. We fix one such (generic) term, and show that it is bounded as $\varepsilon \rightarrow 0$. Such a term can be written as

$$
\begin{aligned}
& \iiint 1_{[0, t]}\left(s_{1}\right) q_{s_{1}}(x) \prod_{i \in U}\left(\left\{\prod_{m \in U_{i}} \mathcal{D}_{-s_{m}}\right\} 1_{[0, t]}\left(\sum_{l \leqslant i} s_{l}\right)\right) q_{s_{i}, \varepsilon}(0) \\
& \quad \times \prod_{j \in V}\left(\left\{\prod_{m \in U_{j}} \mathcal{D}_{-s_{m}}\right\} Q_{\varepsilon}\left(t-\sum_{l<j} s_{l}\right)\right) \delta\left(s_{j}\right) \\
& \quad \times \prod_{k \in W}\left(\left\{\prod_{m \in U_{k}} \mathcal{D}_{-s_{m}}\right\} 1_{[0, t]}\left(\sum_{l \leqslant k} s_{l}\right)\right) q_{s_{k}}(y-x) \prod_{l=1}^{2 n} \mathrm{~d} s_{l} \mathrm{~d} \mu(x) \mathrm{d} \mu(y)
\end{aligned}
$$

where $U=\bigcup_{r} U_{r}$.

For the remainder of this section we restrict our discussion to the case of $\beta<2$. The case of Brownian motion will follow by the same line of reasoning but will be simpler due to the fact that for Brownian motion $u^{1}(x)$ has a logarithmic (as opposed to a power) singularity as $|x| \rightarrow 0$.

Let $v=2 / \beta-1$. Our approach is to bound the $d s_{l}$ integration, proceeding in decreasing order of $l$, and showing that each $\mathcal{D}_{-s_{m}}$ allows us to 'extract' a factor $s_{m}^{\nu(1+\delta)}$, which will then allow us to control $q_{s_{m}}$ near $s_{i}=0$. We must consider in turn the three cases of $l \in V, U, W$. We show how to bound each integration seperately and then use a counting argument to show how to put it all together. As the reader goes through the following lines, he should observe how each $J_{1}^{\varepsilon}$ contributes a factor of $Q_{\varepsilon}(\cdot)$, either directly as $J_{1, \uparrow}^{\varepsilon}$, or indirectly as $J_{1, \downarrow}^{\varepsilon}$ through the operator $\mathcal{D}_{-s \text {. }}$.

Consider first $j \in V$, and use the mean value theorem, monotonicity and $Q(t) \sim$ $C t^{-v}$ to bound

$$
\left|\left\{\prod_{m \in U_{j}} \mathcal{D}_{-s_{m}}\right\} Q_{\varepsilon}\left(t-\sum_{l \leqslant j} s_{l}\right)\right| \leqslant C Q^{1+\left|U_{j}\right|+\delta}\left(t-\sum_{l \leqslant j} s_{l}\right) \prod_{m \in U_{j}} s_{m}^{\nu(1+\delta)} .
$$


Here and throughout the paper $\delta>0$ will denote a constant which may change from line to line but which can be made arbitrarily small. Similarly, $C$ will denote a finite constant which may change from line to line.

Let

$$
u^{0}(x, \rho)= \begin{cases}\left(u^{0}(x)\right)^{\rho}=c|x|^{-(2-\beta) \rho} & \text { if }|x| \leqslant 1 \\ c|x|^{-(2+\beta)} & \text { if }|x| \geqslant 1 .\end{cases}
$$

We note that $C^{\prime} u^{0}(x, 1) \leqslant u^{1}(x) \leqslant C u^{0}(x, 1)$, see Lemma 5 .

We now state two lemmas which will allow us to handle respectively $i \in U$ and $k \in W$. The proofs of these lemmas will be given in the final section. The second display in each lemma will only be used in the following section when we prove Theorem 3 .

LEMMA 3.-

$$
\begin{aligned}
& \int\left|\left\{\prod_{m \in U_{k}} \mathcal{D}_{-s_{m}}\right\} 1_{[0, t]}\left(\sum_{l \leqslant k} s_{l}\right)\right| q_{s_{k}}(y-x) Q^{h_{k}+\delta}\left(t-\sum_{l \leqslant k} s_{l}\right) \mathrm{d} s_{k} \\
& \quad \leqslant C u^{0}\left(y-x, 1+\left|U_{k}\right|+h_{k}+\delta\right) \prod_{m \in U_{k}} s_{m}^{\nu(1+\delta)} .
\end{aligned}
$$

Furthermore, for any $\rho \geqslant 0$ with $\left(\rho+\left|U_{k}\right|+h_{k}\right) v<1$ we have

$$
\begin{gathered}
\int\left|\left\{\prod_{m \in U_{k}} \mathcal{D}_{-s_{m}}\right\} 1_{\left[r_{i-1}, r_{i}\right]}\left(\sum_{l \leqslant k} s_{l}\right)\right| q_{s_{k}}(y-x) Q^{h_{k}+\delta}\left(t-\sum_{l \leqslant k} s_{l}\right) \mathrm{d} s_{k} \\
\quad \leqslant C\left|r_{i}-r_{i-1}\right|^{\rho v} u^{0}\left(y-x, 1+\rho+\left|U_{k}\right|+h_{k}+\delta\right) \prod_{m \in U_{k}} s_{m}^{\nu(1+\delta)} .
\end{gathered}
$$

LEMMA 4. -

$$
\begin{aligned}
& \int\left|\left\{\prod_{m \in U_{i}} \mathcal{D}_{-s_{m}}\right\} 1_{[0, t]}\left(\sum_{l \leqslant i} s_{l}\right)\right| s_{i}^{\nu(1+\delta)} q_{s_{i}, \varepsilon}(0) Q^{h_{i}+\delta}\left(t-\sum_{l \leqslant i} s_{l}\right) \mathrm{d} s_{i} \\
& \quad \leqslant C Q^{\left|U_{i}\right|+h_{i}+\delta}\left(t-\sum_{l<i} s_{l}\right) \prod_{m \in U_{i}} s_{m}^{\nu(1+\delta)} .
\end{aligned}
$$

Furthermore, for any $\rho^{\prime} \geqslant 0$ with $\left(\rho^{\prime}+|\bar{U}|+h\right) v<1$ we have

$$
\begin{gathered}
\int\left|\left\{\prod_{m \in \bar{U}} \mathcal{D}_{-s_{m}}\right\} 1_{\left[r_{i-1}, r_{i}\right]}\left(\sum_{l \leqslant j} s_{l}\right)\right| q_{s_{j}, \varepsilon}(0) Q^{h+\delta}\left(r_{i}-\sum_{l \leqslant j} s_{l}\right) \mathrm{d} s_{j} \\
\leqslant C\left|r_{i}-r_{i-1}\right|^{\rho^{\prime} v} Q^{1+\rho^{\prime}+|\bar{U}|+h+\delta}\left(r_{i-1}-\sum_{l<j} s_{l}\right) \prod_{m \in \bar{U}} s_{m}^{\nu(1+\delta)} .
\end{gathered}
$$

We now explain how to put all these steps together to show that the terms on the right hand side of (2.6) are uniformly bounded in $\varepsilon$. Using (3.4), (3.5) and (3.7) it is easy to check that for each $i \in U$ we pick up a factor $s_{i}^{v(1+\delta)}$ prior to integrating with respect to $d s_{i}$, which allows us to control the singular term $q_{s_{i}, \varepsilon}(0)$. Integrating with respect to $s_{i}, i \in U$ contributes $\left|U_{i}\right|$ factors of $Q_{\varepsilon}$, while each $s_{j}, j \in V$ contributes $1+\left|U_{j}\right|$ factors of $Q_{\varepsilon}$. If $k^{+}$denotes the successor to $k$ in $W$, (with natural ordering), the number of such $Q_{\varepsilon}$ factors arising in this manner from $s_{r}$ with $r$ between $k$ and $k^{+}$is $h_{k}=$ 
$\sum_{k<r<k^{+}}\left\{\left|U_{r}\right|+1_{V}(r)\right\}$. (If $k$ is the largest element in $W$, set $k^{+}=2 n+1$ ). Integrating with respect to $s_{k}, k \in W$ replaces these $h_{k}$ factors of $Q_{\varepsilon}$ by $u^{0}\left(y-x, 1+\left|U_{k}\right|+h_{k}+\delta\right)$. If we use $k_{0}$ to denote the smallest element in $W$, then after integrating with respect to $s_{k_{0}}$ we are left with $\sum_{k \in W}\left(1+\left|U_{k}\right|+h_{k}+\delta\right)$ 'powers' of $u^{0}(y-x)$, i.e.

$$
u^{0}\left(y-x,|W|+\sum_{r \geqslant k_{0}}\left\{\left|U_{r}\right|+1_{V}(r)\right\}+\delta\right) .
$$

Similarly, integrating with respect to $s_{1}$ replaces all factors of $Q_{\varepsilon}$ picked up after integrating with respect to $s_{k_{0}}$ with

$$
u^{0}\left(x, 1+\sum_{r<k_{0}}\left\{\left|U_{r}\right|+1_{V}(r)\right\}+\delta\right) .
$$

Since

$$
|W|+\sum_{r \geqslant k_{0}}\left\{\left|U_{r}\right|+1_{V}(r)\right\}+1+\sum_{r<k_{0}}\left\{\left|U_{r}\right|+1_{V}(r)\right\}=1+|W|+|U|+|V|=2 n,
$$

our assumption that $\sup _{x} \int u^{0}(x-y, 2 n-1+\zeta) \mathrm{d} \mu(y)<\infty$ completes the proof that the terms on the right hand side of (2.6) are uniformly bounded in $\varepsilon$.

\section{Quadratic variation}

We begin by focusing on the case of $\beta<2$. The basic idea which will now be exploited to show that $\gamma_{n}(\mu ; t)$ has zero quadratic variation is that $\operatorname{since} \sup _{x} \int u^{0}(x-y, 2 n-1+$ $\zeta) \mathrm{d} \mu(y)<\infty$, in some sense $d \mu(x) \mathrm{d} \mu(y)$ can 'handle' $2(2 n-1+\zeta)=4 n-2+2 \zeta$ 'powers' of $u^{0}$, and since only $2 n$ powers appear in (3.9)-(3.11), there is an excess capacity to handle an additional $2 n-2+2 \zeta$ powers. We will see that these can be 'traded' for $(2 n-2+2 \zeta) v>1$ powers of $\left|r_{i}-r_{i-1}\right|$. We now provide the details.

Proof of Theorem 3. - As mentioned, we first discuss the case of $\beta<2$. Unraveling the term for a fixed $j$ on the right hand side of (2.9), we obtain expressions similar to (3.3) with the following differences: each factor of the form $1_{[0, t]}\left(\sum_{l \leqslant m} s_{l}\right)$ with $m \geqslant j$ is replaced by $1_{\left[r_{i-1}, r_{i}\right]}\left(\sum_{l \leqslant m} s_{l}\right)$, while each factor of the form $1_{[0, t]}\left(\sum_{l \leqslant m} s_{l}\right)$ with $m<j$ is replaced by $1_{\left[0, r_{i-1}\right]}\left(\sum_{l \leqslant m} s_{l}\right)$, and if $r_{s}(j)=1$ then, see (2.13), the operator $\mathcal{D}_{s_{j}}$ is not present. (Since this operator was essential in controlling the non-integrability of $q_{s_{j}}(0)$ near $s_{j}=0$, we will have to provide a different way to deal with this problem, see below.)

A: Consider the first term on the rhs of (2.9). As mentioned, the analogue of (3.3) has each $1_{[0, t]}$ replaced by $1_{\left[r_{i-1}, r_{i}\right]}$. We proceed as in the previous section except that we apply (3.6) when we integrate with respect to $s_{k_{0}}$ and $s_{1}$. We obtain the bound

$$
\begin{gathered}
\left|r_{i}-r_{i-1}\right|^{\left(\rho+\rho^{\prime}\right) v} u^{0}\left(x, 1+\rho^{\prime}+\sum_{r<k_{0}}\left\{\left|U_{r}\right|+1_{V}(r)\right\}+\delta\right) \\
u^{0}\left(y-x, \rho+|W|+\sum_{r \geqslant k_{0}}\left\{\left|U_{r}\right|+1_{V}(r)\right\}+\delta\right) .
\end{gathered}
$$


By assumptions we can find $\zeta>0$ with $\sup _{x} \int u^{0}(x-y, 2 n-1+\zeta) \mathrm{d} \mu(y)<\infty$ and $(2 n-1+2 \zeta)(2-\beta)>2$. Choose $\rho^{\prime}, \rho$ so that

$$
1+\rho^{\prime}+\sum_{r<k_{0}}\left\{\left|U_{r}\right|+1_{V}(r)\right\}+\delta=2 n-1+\zeta
$$

and

$$
\rho+|W|+\sum_{r \geqslant k_{0}}\left\{\left|U_{r}\right|+1_{V}(r)\right\}+\delta=2 n-1+\zeta .
$$

Using (3.11) now shows that

$$
\rho^{\prime}+\rho=2 n-2+2 \zeta
$$

so that after integrating with respect to $\mathrm{d} \mu(x) \mathrm{d} \mu(y)$ we are left with the bound $\left|r_{i}-r_{i-1}\right|^{(2 n-2+2 \zeta) v}$. Since our assumption that $(2 n-1+2 \zeta)(2-\beta)>2$ implies $(2 n-2+2 \zeta) v>1$. we see that the first term on the rhs of (2.9) contributes zero to the quadratic variation.

B: Consider one of the later terms on the rhs of (2.9). We treat the case that $r_{s}(j)=1$. The case of $r_{s}(j)=2$ is similar. Let $k^{*}=\inf \{k \in W \mid k>j\}$. Assume first that $k^{*}=k_{0}$. As above, we apply we apply (3.6) when we integrate with respect to $s_{k_{0}}$. The $d s_{j}$ integral is our last opportunity to extract powers of $\left|r_{i}-r_{i-1}\right|$. Recall that since $\mathcal{D}_{-s_{j}}$ is absent, we never picked up a factor $s_{j}^{\nu+\delta}$. We use (3.8). As in case A, we can use this to show that our term contributes zero to the quadratic variation.

Assume finally that $k_{0}<k^{*}$. We proceed as before except that we apply (3.6) when we integrate with respect to $s_{k^{*}}$, and (3.8) when we integrate with respect to $s_{j}$. This generates a large number of $Q$ factors that can only be handled by the $d s_{1}$ integration. We thus temporarily interupt our procedure of integrating with respect to $s_{l}$ in decreasing order of $l$, and integrate now with respect to $s_{1}$. We use (3.5) in the form

$$
\begin{gathered}
\int \prod_{\substack{a \in U \\
a<j}}\left|\left\{\prod_{m \in U_{a}} \mathcal{D}_{-s_{m}}\right\} 1_{\left[0, r_{i-1}\right]}\left(\sum_{l \leqslant a} s_{l}\right)\right| \prod_{\substack{b \in V \\
b<j}} Q^{1+\left|U_{b}\right|+\delta}\left(r_{i-1}-\sum_{l \leqslant b} s_{l}\right) \\
\times Q^{1+\rho^{\prime}+\sum_{j \leqslant r<k^{*}}\left\{\left|U_{r}\right|+1_{V}(r)\right\}+\delta}\left(r_{i-1}-\sum_{l<j} s_{l}\right) q_{s_{1}}(x) \mathrm{d} s_{1} \\
\leqslant u^{0}\left(x, 1+\rho^{\prime}+\sum_{r<k^{*}}\left\{\left|U_{r}\right|+1_{V}(r)\right\}+\delta\right) \prod_{\substack{a \in U \\
a<j}} \prod_{m \in U_{a}} s_{m}^{\nu(1+\delta)} .
\end{gathered}
$$

We then resume our procedure of integrating with respect to $s_{l}$ in decreasing order of $l$. The integral with respect to $s_{a}$ for each remaining $a \in U$ is finite, while those with respect to $s_{k}$ for each remaining $k \in W$ each provide a factor of $u^{1}(y-x)$. The case of $k_{0}<k^{*}$ is then completed using (4.2) and (4.3), but with $k_{0}$ replaced by $k^{*}$. This completes the proof that $\gamma_{n}(\mu ; t)$ has zero quadratic variation when $\beta<2$. The case of Brownian motion is even easier, using Lemma 6 in place of Lemma 5 to prove the necessary analogues of Lemmas 3 and 4 , see the next section. Condition (1.12) for some $\zeta>1$ is only needed to extract a factor $\left|r_{i}-r_{i-1}\right|^{\zeta^{\prime}}$ for some $\zeta^{\prime}>1$. 
To complete the proof of our Theorem it only remains to show that we can choose a version of $\left\{\pi_{t}^{\mu, n-1}(A) ;(A, t) \in \mathcal{B}\left(R^{2}\right) \times R_{+}\right\}$which is a.s. a measure in $A$ and continuous in $t$, and such that (1.15) holds. Under the conditions of our Theorem this follows from the methods of [12].

\section{Proofs of Lemmas 3 and 4}

Proof of Lemma 4. - Assume first that $U_{i} \neq \emptyset$.

$$
\begin{aligned}
\int \mid & \left\{\prod_{m \in U_{i}} \mathcal{D}_{-s_{m}}\right\} 1_{[0, t]}\left(\sum_{l \leqslant i} s_{l}\right) \mid s_{i}^{v(1+\delta)} q_{s_{i}, \varepsilon}(0) Q^{h_{i}+\delta}\left(t-\sum_{l \leqslant i} s_{l}\right) \mathrm{d} s_{i} \\
\leqslant & \int\left|\left\{\prod_{m \in U_{i}} \mathcal{D}_{-s_{m}}\right\} 1_{[0, t]}\left(\sum_{l \leqslant i} s_{l}\right)\right| s_{i}^{-1+v \delta} Q^{h_{i}+\delta}\left(t-\sum_{l \leqslant i} s_{l}\right) \mathrm{d} s_{i} \\
\leqslant & Q^{\left|U_{i}\right|+h_{i}+3 \delta}\left(t-\sum_{l<i} s_{l}\right) \\
& \times \int\left|\left\{\prod_{m \in U_{i}} \mathcal{D}_{-s_{m}}\right\} 1_{[0, t]}\left(\sum_{l \leqslant i} s_{l}\right)\right| s_{i}^{-1+\left(\left|U_{i}\right|+h_{i}+4 \delta\right) v} Q^{h_{i}+\delta}\left(t-\sum_{l \leqslant i} s_{l}\right) \mathrm{d} s_{i},
\end{aligned}
$$

where we have used the presence of $\left|\prod_{m \in U_{i}} \mathcal{D}_{-s_{m}} 1_{[0, t]}\left(\sum_{l \leqslant i} s_{l}\right)\right|$ to guarantee that $s_{i} \geqslant t-\sum_{l<i} s_{l}$. We use the generalized Hölder's inequality to bound

$$
\begin{aligned}
& \int\left|\left\{\prod_{m \in U_{i}} \mathcal{D}_{-s_{m}}\right\} 1_{[0, t]}\left(\sum_{l \leqslant i} s_{l}\right)\right| s_{i}^{-1+\left(\left|U_{i}\right|+h_{i}+4 \delta\right) v} Q^{h_{i}+\delta}\left(t-\sum_{l \leqslant i} s_{l}\right) \mathrm{d} s_{i} \\
& \quad \leqslant\left\|\left\{\prod_{m \in U_{i}} \mathcal{D}_{-s_{m}}\right\} 1_{[0, t]}\left(\sum_{l \leqslant i} s_{l}\right)\right\|_{a}\left\|s_{i}^{-1+\left(\left|U_{i}\right|+h_{i}+4 \delta\right) v}\right\|_{b}\left\|Q^{h_{i}+\delta}\left(t-\sum_{l \leqslant i} s_{l}\right)\right\|_{c} \\
& \quad \leqslant C \prod_{m \in U_{i}} s_{i}^{\nu(1+\delta)},
\end{aligned}
$$

where $1 / a=\left(\left|U_{i}\right|+\delta\right) v, 1 / b=1-\left(\left|U_{i}\right|+h_{i}+3 \delta\right) v$, and $1 / c=\left(h_{i}+2 \delta\right) v$. Our choice of $c$ makes $\left\|Q^{h_{i}+\delta}\left(t-\sum_{l \leqslant i} s_{l}\right)\right\|_{c}<\infty$, while our choice of $b$ makes $\left\|s_{i}^{-1+\left(\left|U_{i}\right|+h_{i}+4 \delta\right) v}\right\|_{b}<\infty$. Finally, for each $m \in U_{i}$ we note that $\left\{\prod_{m \in U_{i}} \mathcal{D}_{-s_{m}}\right\}$ $1_{[0, t]}\left(\sum_{l \leqslant i} s_{l}\right)$ is the characteristic function of a finite union of intervals of length $\leqslant s_{m}$ so that we have $\left\|\left\{\prod_{m \in U_{i}} \mathcal{D}_{-s_{m}}\right\} 1_{[0, t]}\left(\sum_{l \leqslant i} s_{l}\right)\right\|_{a} \leqslant C s_{m}^{\left(\left|U_{i}\right|+\delta\right) \nu}$, and the last line of (5.2) follows by interpolation and adjustment of $\delta$.

If, on the other hand, we have $U_{i}=\emptyset$, we use the simple identity for $a+b>1$

$$
\int s^{-a}(t-s)^{-b} \mathrm{~d} s=C t^{-(a+b-1)}
$$

to bound

$$
\int s_{i}^{v(1+\delta)} q_{s_{i}, \varepsilon}(0) Q^{h_{i}+\delta}\left(t-\sum_{l \leqslant i} s_{l}\right) \mathrm{d} s_{i}
$$




$$
=\int s_{i}^{-1+v \delta} Q^{h_{i}+\delta}\left(t-\sum_{l \leqslant i} s_{l}\right) \mathrm{d} s_{i} \leqslant Q^{h_{i}+\delta}\left(t-\sum_{l<i} s_{l}\right)
$$

which completes the proof of (3.7).

To prove (3.8) we begin by using the presence of $1_{\left[r_{i-1}, r_{i}\right]}\left(\sum_{l \leqslant j} s_{l}\right)$ to guarantee that $s_{j} \geqslant r_{i-1}-\sum_{l<j} s_{l}$ we have that

$$
\begin{aligned}
\int \mid & \left\{\prod_{m \in \bar{U}} \mathcal{D}_{-s_{m}}\right\} 1_{\left[r_{i-1}, r_{i}\right]}\left(\sum_{l \leqslant j} s_{l}\right) \mid q_{s_{j}, \varepsilon}(0) Q^{h+\delta}\left(r_{i}-\sum_{l \leqslant j} s_{l}\right) \mathrm{d} s_{j} \\
= & \int\left|\left\{\prod_{m \in \bar{U}} \mathcal{D}_{-s_{m}}\right\} 1_{\left[r_{i-1}, r_{i}\right]}\left(\sum_{l \leqslant j} s_{l}\right)\right| s_{j}^{-1-v} Q^{h+\delta}\left(r_{i}-\sum_{l \leqslant j} s_{l}\right) \mathrm{d} s_{j} \\
\leqslant & Q^{\rho+1+|\bar{U}|+h+4 \delta}\left(r_{i-1}-\sum_{l<j} s_{l}\right) \int\left|\left\{\prod_{m \in \bar{U}} \mathcal{D}_{-s_{m}}\right\} 1_{\left[r_{i-1}, r_{i}\right]}\left(\sum_{l \leqslant j} s_{l}\right)\right| \\
& \times s_{j}^{-1+(\rho+|\bar{U}|+h+4 \delta) v} Q^{h+\delta}\left(r_{i}-\sum_{l \leqslant j} s_{l}\right) \mathrm{d} s_{j} .
\end{aligned}
$$

We use the generalized Hölder's inequality as in (5.2), this time with $1 / a=(\rho+|\bar{U}|+$ $\delta) v, 1-1 / b=(\rho+|\bar{U}|+h+3 \delta) v, 1 / c=(h+2 \delta) v$ to bound

$$
\begin{aligned}
\int & \left|\left\{\prod_{m \in \bar{U}} \mathcal{D}_{-s_{m}}\right\} 1_{\left[r_{i-1}, r_{i}\right]}\left(\sum_{l \leqslant j} s_{l}\right)\right| s_{j}^{-1+(\rho+|\bar{U}|+h+4 \delta) v} Q^{h+\delta}\left(r_{i}-\sum_{l \leqslant j} s_{l}\right) \mathrm{d} s_{j} \\
& \leqslant\left\|\left\{\prod_{m \in \bar{U}} \mathcal{D}_{-s_{m}}\right\} 1_{\left[r_{i-1}, r_{i}\right]}\left(\sum_{l \leqslant j} s_{l}\right)\right\|_{a}\left\|s_{j}^{-1+(\rho+|\bar{U}|+h+4 \delta) v}\right\|_{b}\left\|Q^{h+\delta}\left(r_{i}-\sum_{l \leqslant j} s_{l}\right)\right\|_{c} \\
& \leqslant C\left|r_{i}-r_{i-1}\right|^{\rho v} \prod_{m \in \bar{U}} s_{m}^{\nu(1+\delta)},
\end{aligned}
$$

where we have used $\left\|\left\{\prod_{m \in \bar{U}} \mathcal{D}_{-s_{m}}\right\} 1_{\left[r_{i-1}, r_{i}\right]}\left(\sum_{l \leqslant k} s_{l}\right)\right\|_{a} \leqslant C\left|r_{i}-r_{i-1}\right|^{1 / a}$ and interpolated as before, which completes the proof of (3.8).

Proof of Lemma 3. - We now use the generalized Hölder's inequality to bound

$$
\begin{aligned}
\int & \left|\left\{\prod_{m \in U_{k}} \mathcal{D}_{-s_{m}}\right\} 1_{[0, t]}\left(\sum_{l \leqslant k} s_{l}\right)\right| q_{s_{k}}(y-x) Q^{h_{k}+\delta}\left(t-\sum_{l \leqslant k} s_{l}\right) \mathrm{d} s_{k} \\
& \leqslant\left\|\left\{\prod_{m \in U_{k}} \mathcal{D}_{-s_{m}}\right\} 1_{[0, t]}\left(\sum_{l \leqslant k} s_{l}\right)\right\|_{a}\left\|q_{s_{k}}(x-y)\right\|_{b}\left\|Q^{h_{k}+\delta}\left(t-\sum_{l \leqslant k} s_{l}\right)\right\|_{c}
\end{aligned}
$$

with $1 / a=\left(\left|U_{k}\right|+\delta\right) v, 1 / b=1-\left(\left|U_{k}\right|+h_{k}+3 \delta\right) v$, and $1 / c=\left(h_{k}+2 \delta\right) v$.

Our choice of $c$ makes $\left\|Q^{h_{k}+\delta}\left(t-\sum_{l \leqslant k} s_{l}\right)\right\|_{c}<\infty$, while, by Lemma 5 below, our choice of $b$ gives the bound $\left\|q_{s_{k}}(x-y)\right\|_{b} \leqslant C u^{0}\left(y-x, 1+\left|U_{k}\right|+h_{k}+\delta\right)$, where we have readjusted $\delta$ for ease of notation. Handling the term $\|\left\{\prod_{m \in U_{k}} \mathcal{D}_{-s_{m}}\right\} 1_{[0, t]}\left(\sum_{l \leqslant k} s_{l} \|_{a}\right.$ as above now completes the proof of (3.5).

The proof of (3.6) is similar, except that we now take $1 / a=\left(\rho+\left|U_{k}\right|+\delta\right) v$, $1 / b=1-\left(\rho+\left|U_{k}\right|+h_{k}+3 \delta\right) v$, and $1 / c=\left(h_{k}+2 \delta\right) v$ and use the fact that 
$\left\|\left\{\prod_{m \in U_{k}} \mathcal{D}_{-s_{m}}\right\} 1_{\left[r_{i-1}, r_{i}\right]}\left(\sum_{l \leqslant k} s_{l}\right)\right\|_{a} \leqslant C\left|r_{i}-r_{i-1}\right|^{1 / a}$. Interpolating as before completes the proof of (3.6) and hence of Lemma 3.

LEMMA 5. - If $\rho v<1$ and $1 / a=1-\rho v$ then for any $T<\infty$

$$
C_{T}^{\prime} u^{0}(x, 1+\rho) \leqslant\left(\int_{0}^{T}\left(p_{s}(x)\right)^{a} \mathrm{~d} s\right)^{1 / a} \leqslant C_{T} u^{0}(x, 1+\rho)
$$

for some $0<C_{T}^{\prime} \leqslant C_{T}<\infty$ where $p_{s}(x)$ denotes the transition density for the symmetric stable process of order $1<\beta<2$ in $R^{2}$. In particular, for some $C>0$ we have

$$
C u^{0}(x, 1) \leqslant u^{1}(x) .
$$

Proof. - We note the scaling property

$$
p_{s}(x)=\frac{1}{s^{2 / \beta}} p_{1}\left(x / s^{1 / \beta}\right)
$$

and the asymptotics, [3],

$$
p_{1}(x) \sim \frac{C}{|x|^{2+\beta}}, \quad|x| \rightarrow \infty .
$$

Letting $v$ denote a unit vector in $R^{2}$, and using (5.9) we have

$$
\begin{aligned}
\left(\int_{0}^{T}\left(p_{s}(x)\right)^{a} \mathrm{~d} s\right)^{1 / a} & =\left(\int_{0}^{T}\left(\frac{1}{s^{2 / \beta}} p_{1}\left(x / s^{1 / \beta}\right)\right)^{a} \mathrm{~d} s\right)^{1 / a} \\
& =\left(\int_{0}^{T} \frac{1}{s^{2 a / \beta}}\left(p_{1}\left(x / s^{1 / \beta}\right)\right)^{a} \mathrm{~d} s\right)^{1 / a} \\
& =\left(\frac{1}{|x|^{2 a-\beta}} \int_{0}^{T /|x|^{\beta}} \frac{1}{s^{2 a / \beta}}\left(p_{1}\left(v / s^{1 / \beta}\right)\right)^{a} \mathrm{~d} s \mathrm{~d} y\right)^{1 / a} \\
& =\operatorname{Cr}(T, x, a, \beta) \frac{1}{|x|^{2-\beta / a}} \\
& =C r(T, x, a, \beta) \frac{1}{|x|^{2-\beta+\beta \rho v}} \\
& =C r(T, x, a, \beta) \frac{1}{|x|^{(2-\beta)(1+\rho)}}
\end{aligned}
$$

where

$$
r(T, x, a, \beta)=\left(\int_{0}^{T /|x|^{\beta}} \frac{1}{s^{2 a / \beta}}\left(p_{1}\left(v / s^{1 / \beta}\right)\right)^{a} \mathrm{~d} s\right)^{1 / a}
$$




$$
\leqslant\left(\int_{0}^{\infty} \frac{1}{s^{2 a / \beta}}\left(p_{1}\left(v / s^{1 / \beta}\right)\right)^{a} \mathrm{~d} s\right)^{1 / a}<\infty
$$

using (5.10), and $r(T, x, a, \beta) \geqslant c>0$ uniformly for $|x| \leqslant 1$.

This implies our Lemma for $|x| \leqslant 1$. For $|x| \geqslant 1$, (5.9) and (5.10) imply that

$$
p_{s}(x) \leqslant \frac{c_{T}}{|x|^{2+\beta}}, \quad s \leqslant T
$$

and

$$
p_{s}(x) \geqslant \frac{c_{T}^{\prime}}{|x|^{2+\beta}}, \quad T / 2 \leqslant s \leqslant T
$$

so that (5.7) for $|x| \geqslant 1$ follows immediately. This completes the proof of Lemma 5.

We have the following analogue for Brownian motion.

LEMMA 6. - If $a>1$ then for any $T<\infty$

$$
\left(\int_{0}^{T}\left(p_{s}(x)\right)^{a} \mathrm{~d} s\right)^{1 / a} \leqslant \begin{cases}C_{T}|x|^{-2(1-1 / a)} & \text { if }|x| \leqslant 1 \\ C_{T} \exp \left(-x^{2} / 4 T\right) & \text { if }|x| \geqslant 1\end{cases}
$$

where $p_{s}(x)=(2 \pi s)^{-1} \exp \left(-x^{2} / 2 s\right)$ denotes the transition density for Brownian motion in $R^{2}$.

Proof. - This follows from

$$
\begin{aligned}
& (2 \pi)^{-1}\left(\int_{0}^{T} \frac{\exp \left(-a x^{2} / 2 s\right)}{s^{a}} \mathrm{~d} s\right)^{1 / a} \\
& \quad=(2 \pi)^{-1} \exp \left(-a x^{2} / 4 T\right)\left(\int_{0}^{T} \frac{\exp \left(-a x^{2} / 4 s\right)}{s^{a}} \mathrm{~d} s\right)^{1 / a} \\
& \quad \leqslant(2 \pi)^{-1} \frac{\exp \left(-a x^{2} / 4 T\right)}{|x|^{2(1-1 / a)}}\left(\int_{0}^{\infty} \frac{\exp (-a / 4 s)}{s^{a}} \mathrm{~d} s\right)^{1 / a} .
\end{aligned}
$$

\section{Acknowledgement}

I would like to thank M. Yor for some very helpful conversations.

\section{REFERENCES}

[1] Bass R., Khoshnevisan D., Intersection local times and Tanaka formulas, Ann. Inst. H. Poincaré Probab. Stat. 29 (1993) 419-452. 
[2] Bertoin J., Applications des processes de Dirichlet aux temps locaux et temps locaux d'intersection d'un mouvement Brownien, Probab. Theory Related Fields 80 (1989) 433460.

[3] Blumenthal R., Getoor R., Some theorems on stable proceses, Trans. Amer. Math. Soc. 95 (1960) 261-273.

[4] Dynkin E.B., Self-intersection gauge for random walks and for Brownian motion, Ann. Probab. 16 (1988) 1-57.

[5] Fitzsimmons P., Salisbury T., Capacity and energy for multiparameter Markov processes, Ann. Inst. H. Poincaré Probab. Stat. 25 (1989) 325-350.

[6] Föllmer H., Dirichlet processes, in: Proceedings Durham 1980 (Berlin), Lecture Notes Math., Vol. 851, Springer-Verlag, Berlin, 1981, pp. 476-478.

[7] Le Gall J.-F., Proprietes d'intersection des marches aleatoires, I, Comm. Math. Phys. 104 (1986) 471-507.

[8] Le Gall J.-F., Fluctuation results for the Wiener sausage, Ann. Probab. 16 (1988) 991-1018.

[9] Le Gall J.-F., Some properties of planar Brownian motion, Ecole d'ete de probabbilities de St. Flour XX, 1990 (Berlin), Lecture Notes in Mathematics, Vol. 1527, Springer-Verlag, Berlin, 1992.

[10] Le Gall J.-F., Rosen J., The range of stable random walks, Ann. Probab. 19 (1991) 650-705.

[11] Marcus M., Rosen J., Renormalized self-intersection local times and Wick power chaos processes, Mem. Amer. Math. Soc. 142 (675) (1999).

[12] Rosen J., Joint continuity and a Doob-Meyer type decomposition for renormalized intersection local times, Ann. Inst. H. Poincaré Probab. Stat. 35 (1999) 143-176.

[13] Rosen J., Continuity and singularity of the intersection local time of stable processes in $\mathbf{R}^{2}$, Ann. Probab. 16 (1988) 75-79.

[14] Rosen J., The asymptotics of stable sausages in the plane, Ann. Probab. 20 (1992) 29-60.

[15] Rosen J., Joint continuity of renormalized intersection local times, Ann. Inst. H. Poincaré Probab. Stat. 32 (1996) 671-700.

[16] Varadhan S.R.S., Appendix to Euclidian quantum field theory by K. Symanzyk, in: Jost R. (Ed.), Local Quantum Theory, Academic Press, 1969. 